\title{
REAL-TIME STABILITY AND PROFILE COMPARISON MEASUREMENTS BETWEEN TWO DIFFERENT LTPS ${ }^{*}$
}

\author{
Shinan Qian \\ Brookhaven National Laboratory \\ Upton, NY 11973-5000 \\ Duan Jen Wang \\ National Synchrotron Radiation Research Center \\ Hsinchu, Taiwan
}

July, 2005

"This manuscript has been authored by Brookhaven Science Associates, LLC under Contract No. DE-AC02-98CH10886 with the U.S. Department of Energy. The United States Government retains, and the publisher, by accepting the article for publication, acknowledges, a world-wide license to publish or reproduce the published form of this manuscript, or allow others to do so, for the United States Government purposes. 


\title{
Real-time stability and profile comparison measurements between two different LTPs
}

\author{
Shinan Qian \\ Brookhaven National Laboratory, Upton, NY 11973 \\ Duan Jen Wang \\ National Synchrotron Radiation Research Center, Hsinchu, Taiwan
}

\begin{abstract}
The Long Trace Profiler (LTP) is a precise angle measurement instrument, with a sensitivity and accuracy that can be in the sub-micron radian range. LTP characteristics depend on the particular LTP system schematic design, and the quality of components and assembly. The conditions of temperature, alignment, and mirror support during the measurement proccess vary between different laboratories, which influences significantly the test repeatability and accuracy. In this paper we introduce a direct comparison method to test the same object at the same point in the same environment at the same time by using two LTPs, which significantly increases the reliability of the comparison. A compact, portable LTP (PTLTP), which can be carried to different laboratories around the world, is used for comparison testing. Stability comparison experiments between the LTP II at the National Synchrotron Radiation Research Center (NSRRC), and the PTLTP of Brookhaven National Laboratory (BNL) reveal significant differences in performance between the instruments. The experiment is set up so that each optical head simultaneously records both its own sample probe beam and also the probe beam from the other optical head. The two probe beams are reflected from same point on the mirror. Tests show that the stability of the PTLTP with a monolithic beam splitter is 10 times better than the stability of the LTP II which has a separated beam splitter unit. A scheme for comparing scanning measurements of a mirror is introduced. Experimental results show a significant difference between the two LTPs due mainly to distortions in the optical components inside the optical head. A new scheme is proposed for further mirror comparison scanning tests.
\end{abstract}

\section{INTRODUCTION}

The Long Trace Profiler is a precise angle measurement instrument, with a sensitivity and accuracy that can be in the sub-micro-radian range. The characteristics of each LTP depend on its particular system schematic, and on the quality of internal components and assembly. Temperature, alignment, and mirror support methods during testing vary between different laboratories, which influences significantly the repeatability and accuracy of the measurement. In order to know actual LTP characteristics, several comparisons are necessary. First, a precise mirror with a calibrated radius of curvature of 10.167 meters is used to calibrate the slope angle calibration coefficient of the LTP. The second method is to compare the angles produced by standard precision instruments (small angle generator, goniometry instrument, sine bar and so on) with those measured by the LTP at the same time, This is a direct comparison/calibration method. For example, at Synchrotrone Trieste in Italy, a 0.1 arc second accuracy theodolite was used as a small angle generator. The third method used is to transport a calibration mirror to different laboratories around the world for testing by different LTPs, then compare the test results. This is an indirect comparison method because the measuring conditions may be different at the different places and times. In this paper we will introduce another real-time direct comparison measurement method between different LTPs.

A portable LTP (PTLTP) [1] developed at BNL can be carried to different laboratories around world for comparison testing. Tests by two LTPs can then be done on the same objects at the same point at the same time in the same environment, even being aligned by the same person. Tests done under these conditions are very reliable. Stability comparison experiments between the LTP II [2] of NSRRC (TWLRP) and the PTLTP of BNL reveal significant performance differences. Sample beams are sent individually from the TWLTP and the PTLTP optical heads, are reflected from the same point on the mirror, and are returned to the TWLTP and PTLTP. Tests show that the stability of the PTLTP with a monolithic beam splitter is almost 10 times better than the stability of the LTP II with separated beam splitter unit. A scheme for comparison measurement on mirror is introduced. 


\section{COMPARISON TEST CONFIGURATION OF TWO LTPS}

One LTP to be compared is the LTP II (Fig 1 b)), a commercial LTP II from Continental Optical Corporation, which is set on a granite table in the optical metrology laboratory of NSRRC in a well temperature-controlled clean room. For simplicity we call it the TWLTP. The LTP II has the following characteristics: a He-Ne laser coupled into a fiber which transfers light to a fiber collimator, producing a parallel pencil beam; a separated equal optical path beam splitter [3], which consists of two right angle prisms and one cube beam splitter to create two parallel pencil beams with a separation about $1 \mathrm{~mm}$ (adjustable); a single lens of focal length $1250 \mathrm{~mm}$ used as a Fourier Transform lens; Princeton Instrument linear array detector with total 1024 pixels of dimensions $25 \times 2500 \mu \mathrm{m}$ and a 15 bit ADC. The LTP II optical head is fixed and is used with a scanning penta-prism LTP [4]. Operating software for the LTP II was provided by Continental Optical.

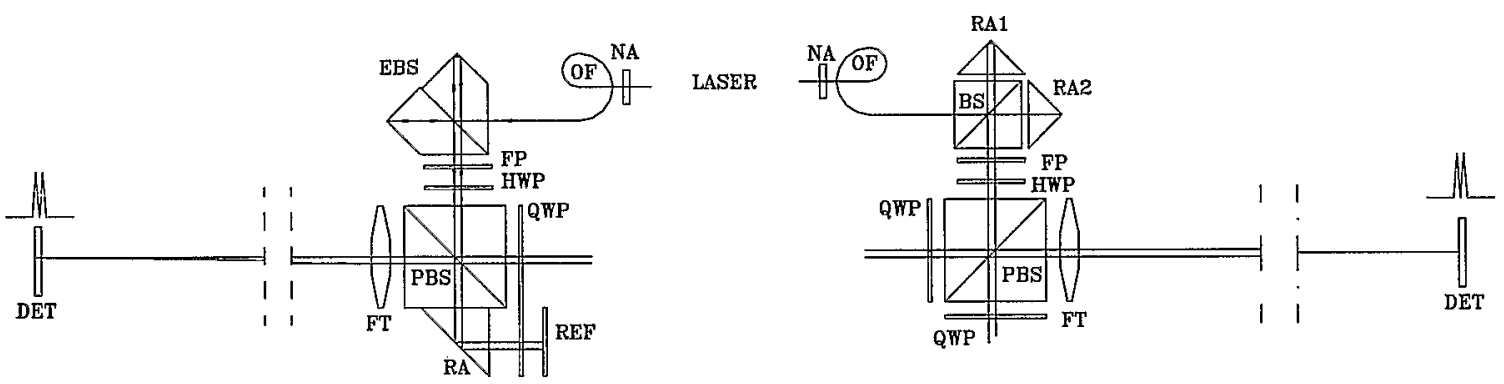

a) Optical schematic of the portable LTP (PTLTP)

b) Optical schematic of the LTP II

Fig. 1: Two optical systems to be compared: (a) PTLTP and (b) LTP II (TWLTP).

The other LTP used in the comparison is a PTLTP (Fig 1a), made at BNL, which was carried to NSSRC for the realtime comparison test. The PTLTP is set on the same granite table together with the LTP II. The PTLTP has the following characteristics: a He-Ne laser is coupled into a fiber to transfer light to a fiber collimator, producing a pencil beam for the PTLTP; an equal optical path beam splitter (EBS) [5, 6], which is a monolithic beam splitter that creates two parallel beams with a separation about $0.8 \mathrm{~mm}$ (not adjustable); a single lens with a focal length $750 \mathrm{~mm}$ (consider

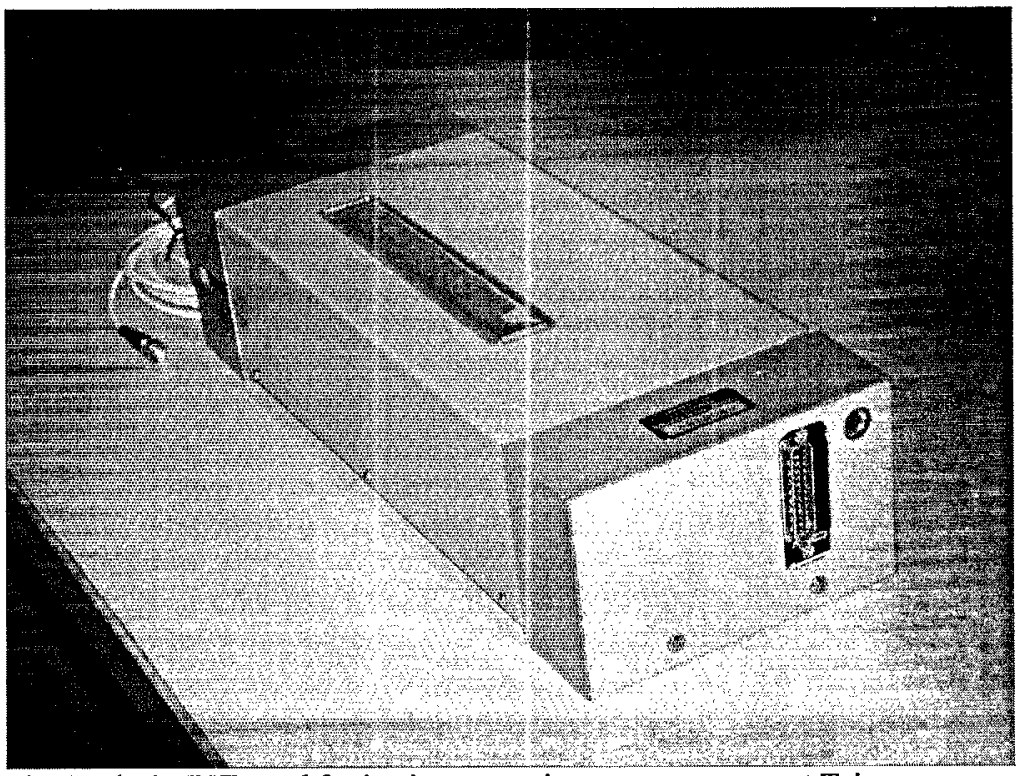

Fig. 2: The PTLTP used for in-situ comparison measurements at Taiwan this LTP only for plane mirror test) used as a Fourier Transform lens; a Cronin linear array CCD camera with a total of 1024 pixels with dimensions of $25 \times 2500$ $\mu \mathrm{m}$ and 16 bit ADC. The PTLTP optical head is also fixed to the table and is used as in the penta-prism scanning mode [4] It uses modified LTP II operating software in order to operate the Cronin CCD. Fig. 2 is a photo of the PTLTP used for the comparison test.

The configuration of the real-time comparison tests between the two LTPs is shown on Fig. 3. This configuration is designed to compare tests of stability and mirror profiles. In order to eliminate the influence produced by slide pitch error, a penta-prism combination unit (PCU) was designed (Fig 3). The PCU directs the sample beam from each optical head to 
the same test point on the mirror under test (MUT). The PCU consists of two penta prisms (PP1 and PP2) and a lateral displacement beam splitter (LDBS), shown inside the circle in Fig. 3. During the scan test, both optical heads are stationary and the PCU is scanned by the slide.

With the PCU, the beam sent from TWLTP travels through PP1 and through LDBS to the MUT, while the beam sent from PTLTP travels through PP2 and then reflected by the LDBS to coincide with the TWLTP beam. The two beams reach the same point on MUT and are reflected back. Each return beam will be split by the LDBS into two beams that each propagate back to the TWLTP and PTLTP. The final result is that each optical head records two fringe patterns from the MUT, one produced by itself and the other by the other optical head. Four scans data files are recorded at the same time. Note that the standard LTP II reference beam is not used in this configuration.

Both the PTLTP beam and the TWLTP beam are reflected an even number of times ( 4 and 8 respectively) along the optical path outside each optical head, so any PCU error rotation, caused by pitch, will not create angle direction variation to the MUT. Since both of the LTPs are operated in the penta-prism scanning mode, no reference correction is necessary.

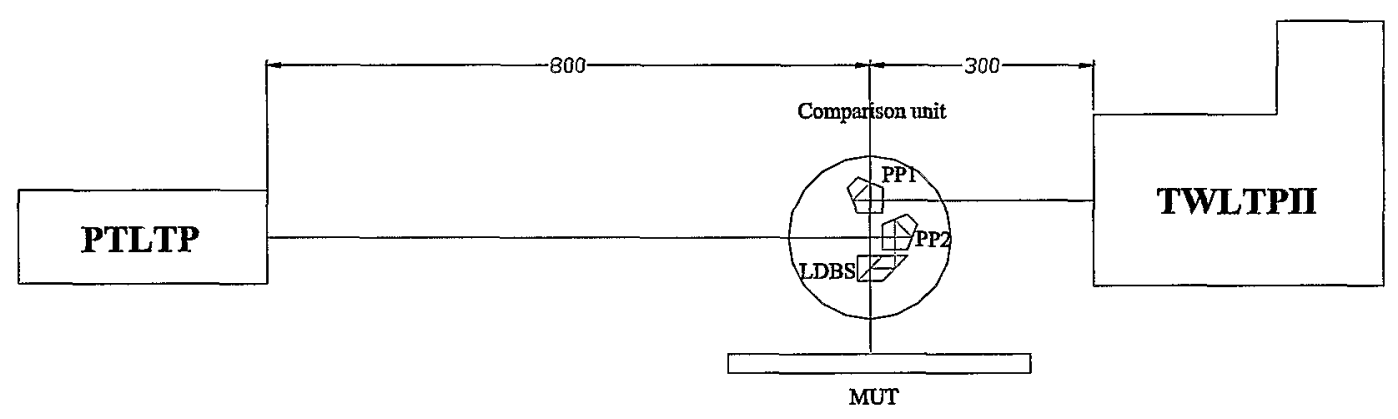

Fig. 3 Configuration of the simultaneous comparison test between two LTPs. The PCU unit is shown inside the circle.

\section{STABUITY COMPARISON TEST}

The stability comparison test is done by starting the stability scans of the two LTPs at the same time. However, if the beams from both LTPs are aligned to be exactly colinear, the two patterns on each detector will overlap, causing severe distortion of the fringes. With this alignment, it would be impossible to make a test. So the PTLTP beam must be tilted slightly to separate the two sets of fringes on each detector. This slight misalignment will not influence the stability test results.

The LTPs were located in a temperature-controlled room without a tent. The stability test lasted for 9 hours overnight. During the test, a precise thermometer with $0.01^{\circ} \mathrm{C}$ resolution was used to monitor the room temperature. As the previous record of NSRRC the room temperature variation is usually well controlled to under $\pm 0.1^{\circ}$. However, during this night, due to an unknown control system disorder, the temperature oscillation was much larger in the range of $\pm 0.25^{\circ}$ (Fig. 4). Fortunately, in two periods of 1 and 1.5 hours at the beginning, the temperature stability was within $\pm 0.1^{\circ}$. As a matter of fact, this incident was a much better test for the stability comparison purpose. We observed dramatic and very interesting results.

Fig. 5 shows the four stability scans for each of the separate beams. Each shows the effect of temperature oscillations of $\pm 0.25^{\circ} \mathrm{C}$ over the 9 hour time period. Two data scans are recorded on the TWLTP: the.TWLTP to TWLTP beam and the PTLTP to TWLTP beam. Two data scans are recorded on the PTLTP: the PTLTP to PTLTP beam and the TWLTP to PTLTP beam. Fig. 6 is an isolated view of only the PTLTP data. In order to clearly see small effects between each component scan, we have inverted one of the scans. 


\section{Temperature during comparison test in SRRC}

(20516TW.tem, 5/16/02/22:46-5/17/02/07:46)

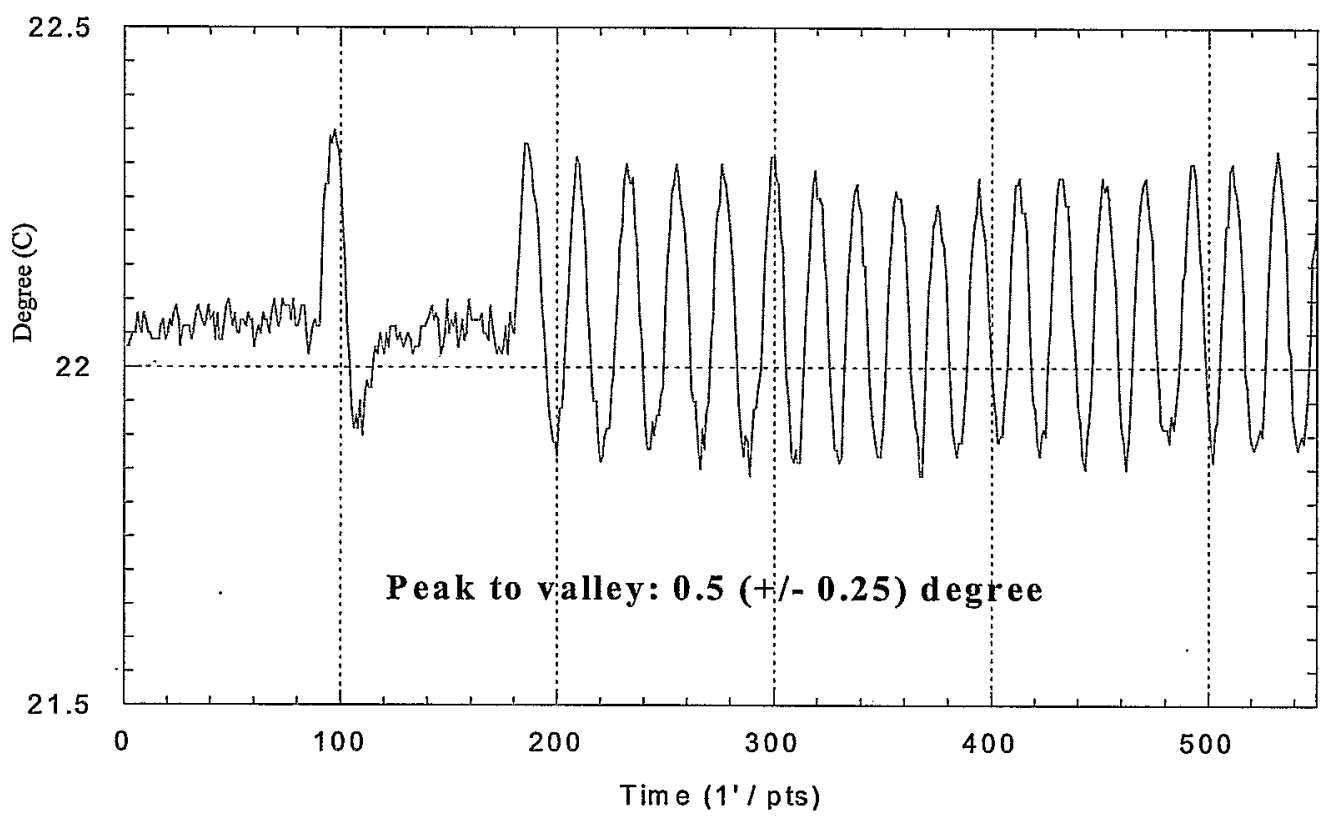

Fig. 4 Temperature oscillations during the stability scan, recorded by Testo thermometer

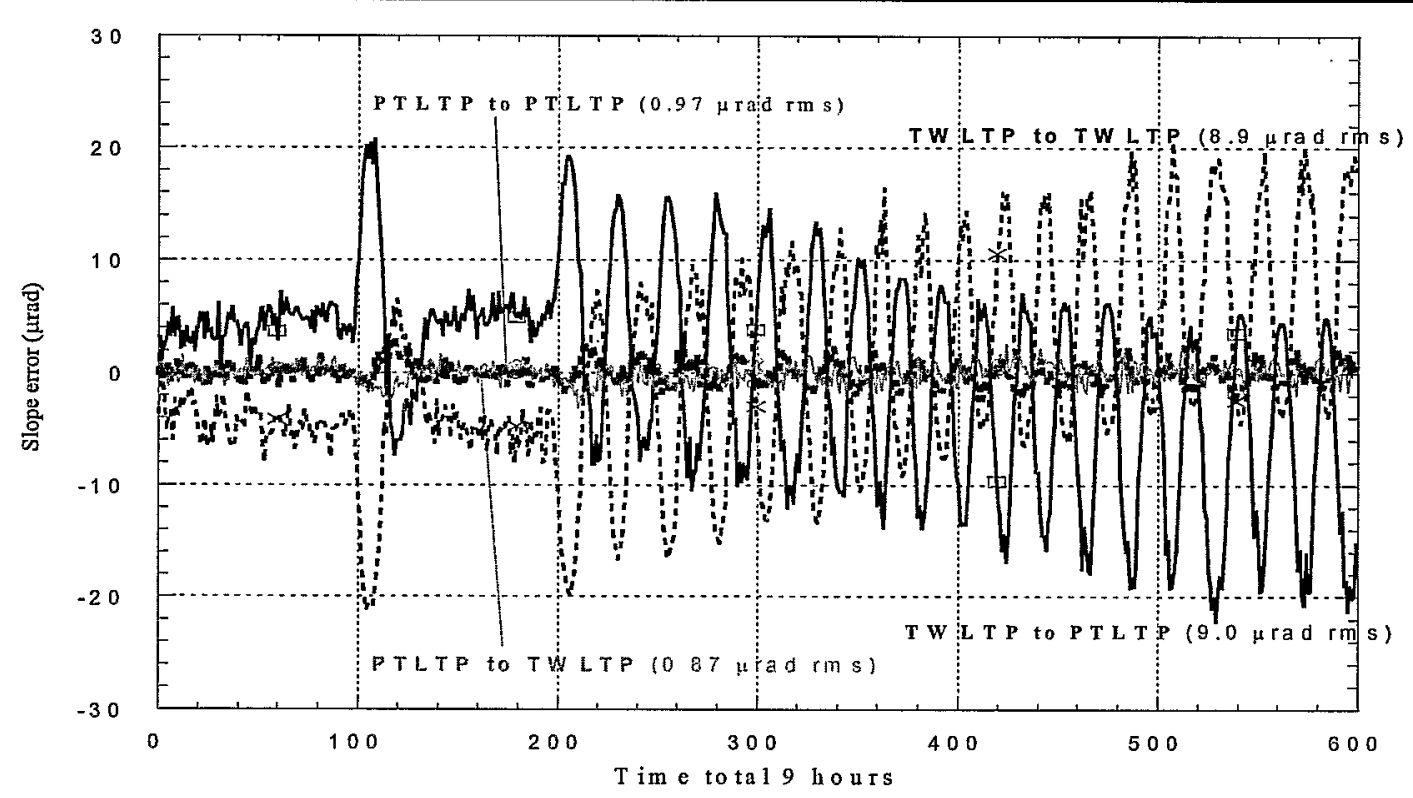

Fig. 5 Stability comparison test on TWLTP and PTLTP 


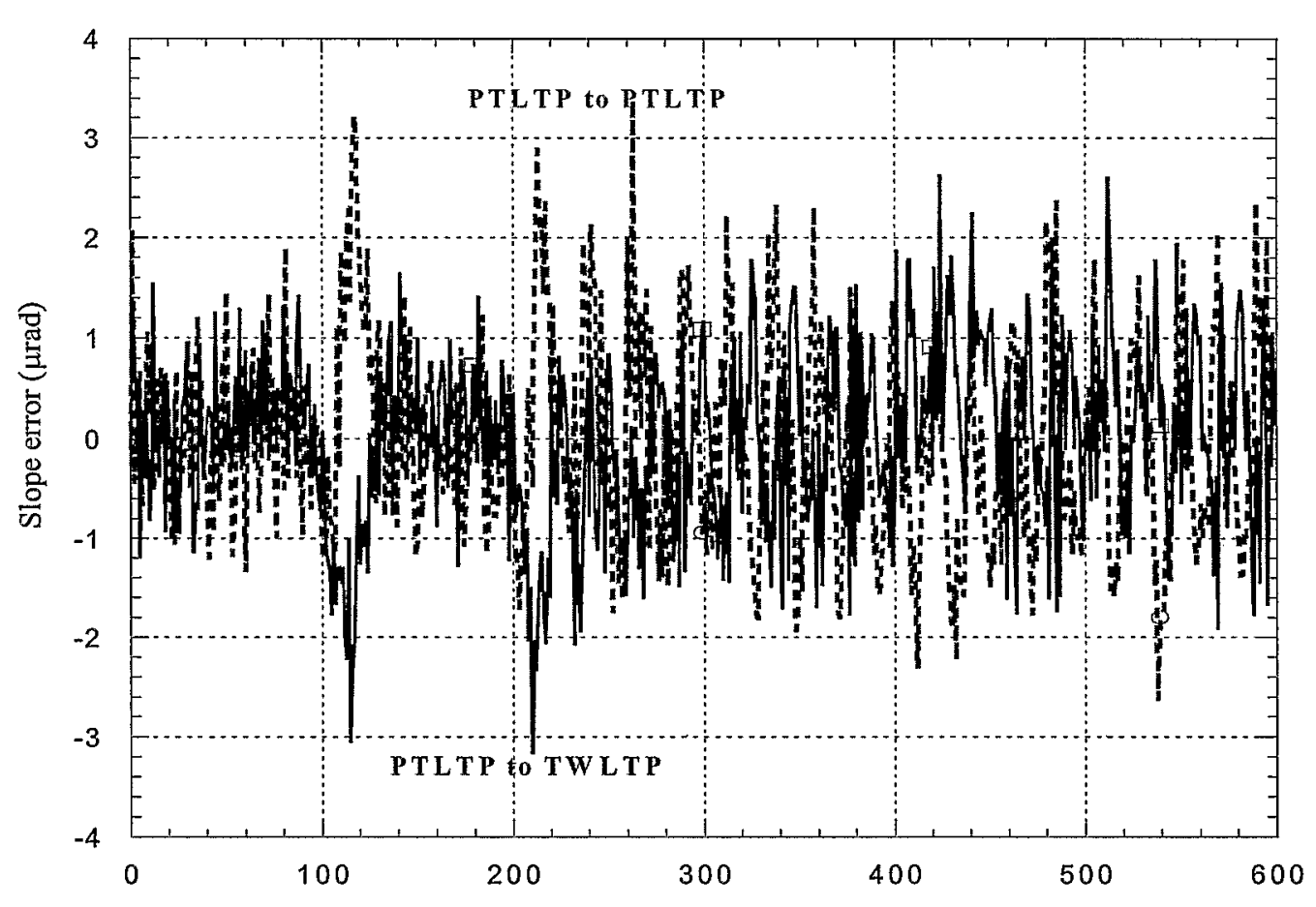

Time total 9 hours

Fig. 6 Stability scan comparison test showing only the PTLTP data.

From the experiment results (Fig.4-6) we can make the following conclusions:

1. The PTLTP is much more stable than the LTP II (TWLTP).

a. The PTLTP is almost 10 times more stable than the LTP $\Pi$ in a room with temperature oscillations of $\pm 0.2^{\circ} \mathrm{C}$. The LTP II produced $9 \mu \mathrm{rad} \mathrm{rms}$ of slope error while PTLTP only produced $0.95 \mu \mathrm{rad} \mathrm{rms}$ of slope error (Fig. 4-6, after 3 hours)

b. The PTLTP is twice as stable than the LTP II in a room with temperature oscillations of $\pm 0.05^{\circ} \mathrm{C}$ over a one hour period. The LTP II produced less than $1 \mu \mathrm{rad}$ rms slope error while PTLTP produced less than $0.5 \mu \mathrm{rad}$ rms slope error (Fig. 4-6, first 1.5 hours)

2. The LTP II is (but the PTLTP is not) the main source of instability because, if the beam is sent from LTP II, the stability is always bad, regardless of whether it is received by the PTLTP or the LTP $\Pi$, and also because, if the beam is sent from the PTLTP, the stability is always good, regardless of whether it is received by the PTLTP or the LTP II.

3. Room temperature oscillation produces a significant LTP II instability. The slope curve oscillation (period, amplitude) exactly coincides with the temperature oscillation curve.

4. High precision measurement requires a highly stable temperature condition. If a sub-microradian measurement is necessary over a long period, the LTP should be maintained in an environment of better than $\pm 0.1^{\circ} \mathrm{C}$.

5. In the LTP II, temperature related instability error is mainly produced in the section of optical system between the laser and the PBS (laser, optical fiber, beam splitter, polarizer and wave plate), and is not produced in the section between the PBS and the CCD, because when the LTP II accepts the beam from PTLTP, the result is stable. Note that the LTP II uses a fiber collimator, which eliminates laser pointing error from the system. The thermal source of LTP II laser is separated from the optical head, so we can conclude that the instability is from beam splitter alone. The monolithic equal optical path prism beam splitter (and equivalently a phase shift beam splitter) used in the PTLTP is much more stable than is a separated equal optical path beam splitter as is used in the LTP II.

6. The LTP II slope curve has a tendency to drift over a long time period, but the PTLTP does not show such a drift. This probably is produced by mechanical relaxation within the LTP II. So the instability in the LTP II 
will be much larger than the $1 \mu \mathrm{rad}$ described above in $1 . \mathrm{b}$ over the 9 hours even without temperature oscillation.

7. The sensitivity and noise level of the two cameras and controllers of the LTP II and the PTLTP are about the same because the noise in the data of PTLTP to PTLTP and PTLTP to TWLTP scans are similar. These scans originate from the PTLTP but are sent to different cameras. This is pointing at detector system.

8. But the noise level of the PTLTP is better than that of the LTP II. Referring to the curves in the period of stable temperature, if the beam is sent from the TWLTP, it will always have larger noise (TWLTP to TWLTP and TWLTP to PTLTP) then that sent from the PTLTP. This is pointing at LTP system.

\section{PRELIMINARY COMPARISON TEST OF A PLANE MIRROR PROFILE}

We used the scanning PCU to make a profile scanning comparison test on a plane mirror. Four files will result from one scan with the two LTPs: each LTP receives two fringe sets, one originating from its own beam and the other originating from the other LTP beam. For the same reason described above, the PTLTP was tilted by a small angle in order to avoid

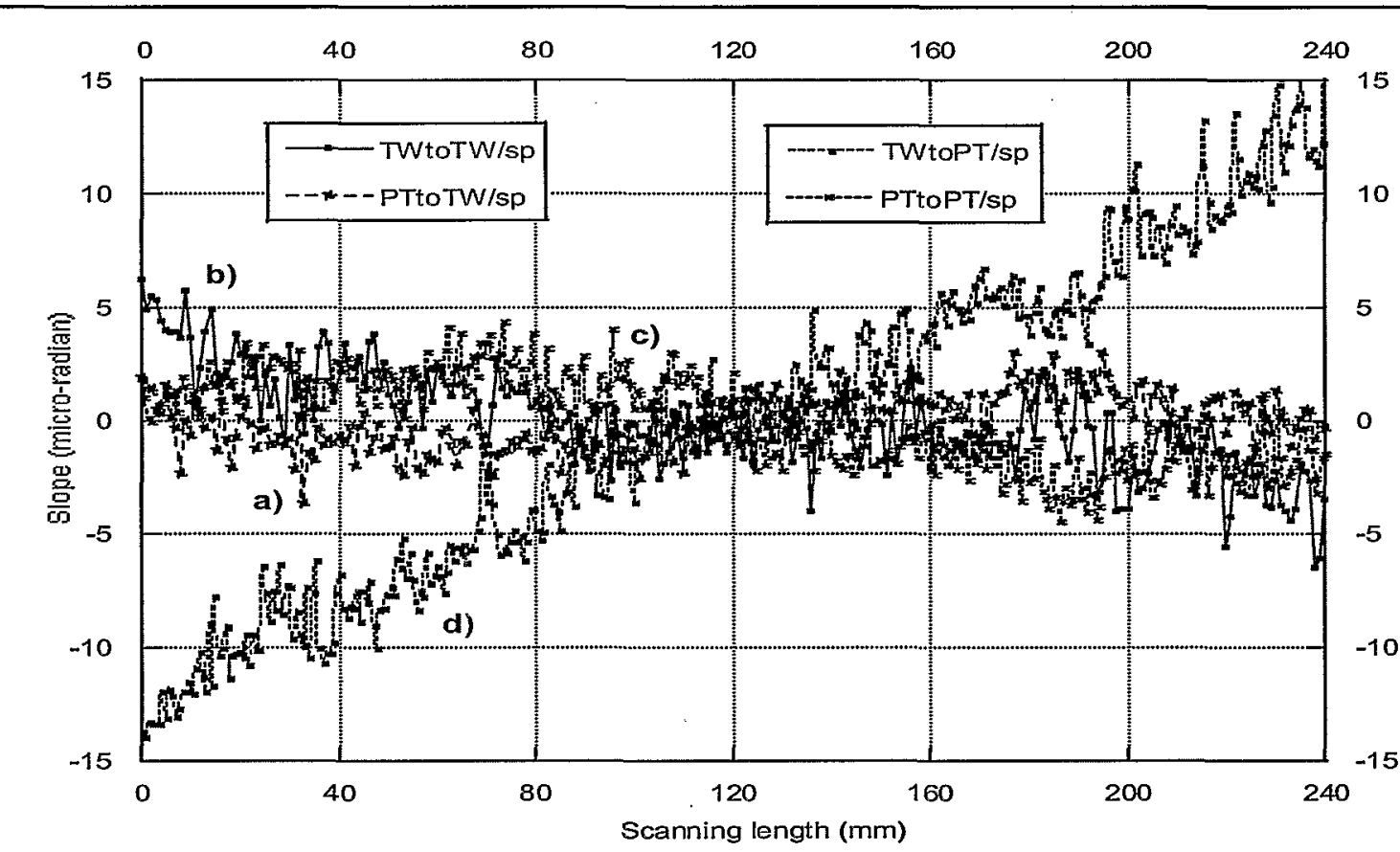

Fig. 7 Preliminary comparison test of a plane mirror profile

the fringe overlapping problem. We performed the test by making a linear scan with the TWLTP while running the PTLTP is the stability scan mode. The two LTPs are started at the same instant manually and are run over the same time period. There is no synchronization signal between the two LTPs over this time. The test result is shown in Fig. 7. Scans (a) and (b) are the slopes measured by the TWLTP with beams originating from the PTLTP and TWLTP respectively, while scans (c) and (d) are the slopes measured by the PTLTP with beams originating from the PTLTP and TWLTP respectively. We can see that there are significant differences between the measurements recorded by these two LTPs.

From this test we find that four test results are very different. Even the scans that appear to be close together do not satisfy the criteria for the precise plane mirror test.

Theoretically, a scan made by use of a penta-prism should be accurate, but the penta-prism must be aligned properly. Lack of accuracy in this case is mainly produced by the tilts of the probe beam away from the optical axis direction, which increases the LTP systematic error. The tilted beams will move laterally over the optical surfaces in the optical system during the scan and pick up phase shifts from the imperfect components, which will be added to the intrinsic 
error of the test surface [7]. Also, because of the tilt in the beam, beams further from center of the FT lens aperture will contain errors produced by focus errors and FT lens aberration. As can be seen in Fig. 3, the $800 \mathrm{~mm}$ distance between the PTLTP and MUT is much larger than the $300 \mathrm{~mm}$ distance between the TWLTP and MUT. This makes the lateral movement in the PTLTP aperture much larger than in the TWLTP. From the geometry of the test configuration we can retrieve the actual test conditions of the four returning beams roughly as reference:

\begin{tabular}{|c|c|c|c|}
\hline Beam from and to & $\begin{array}{c}\text { Angle of returning beam } \\
\text { to receiving LTP (rad) }\end{array}$ & LTP to MUT distance & Beam lateral movement \\
\hline TWLTP to TWLTP & 0.0028 & $300 \mathrm{~mm}$ & $0.84 \mathrm{~mm}$ \\
\hline PTLTP to TWLTP & 0.0001 & $300 \mathrm{~mm}$ & $0.02 \mathrm{~mm}$ \\
\hline TWLTP to PTLTP & 0.0037 & $800 \mathrm{~mm}$ & $2.99 \mathrm{~mm}$ \\
\hline PTLTP to PTLTP & 0.0011 & $800 \mathrm{~mm}$ & $0.91 \mathrm{~mm}$ \\
\hline
\end{tabular}

This can probably explain why the curve of TWLTP to PTLTP is much worse and the curve of PTLTP to TWLTP is much better.

From the experiment results (Fig. 7) we can get some helpful conclusions as following:

1. For profile scanning test on a plane mirror in optical scanning mode, it should set the MUT closer to the LTP in order to reduce the systematic error, because the reference beam has to be set with a tilt angle.

2. For profile scanning test on a plane mirror, it is better to align the returning beam along the optical axis to avoid the lateral movement. A penta-prism scan mode is preferred

3. In order to make precise comparison test to get four tested profiles at the same scan, the two LTPs should not be aligned with an angle. In order to reach this goal, non-tilt and no fringe overlapping solution should apply.

\section{FURTHER CONSIDERATION FOR PROFILE COMPARISON TEST ON A PLANE MIRROR}

In order to keep the four beams parallel to the optical axis, we consider not tilting the PTLTP but changing the software, hardware, and comparison scheme. The principle is to take the fringe data in a time sequence, one by one quickly at each scan position. It means the fringes are really overlapping in position on the detector but are not overlapping at the same time on the detector. The practical example could use a mechanical or electrical switch in each LTP beam to close one laser beam while the other LTP switch is open for data acquisition. Then the switches are reversed for second LTP data acquisition. In order to execute this option, the LTP software control system should be modified to operate the data acquisitions and synchronization of the two LTPs. We expect the further experiment will have high accuracy test result.

\section{CONCLUSION}

Real-time comparison measurements between different LTPs are very helpful for investigating stability, accuracy, noise level, structure, system and different characteristics of the LTP. A portable LTP is necessary for this purpose. The main advantage of real-time comparison is: tests of two LTPs can be done on the same mirror at the same point at the same time in the same environment, even with alignment done by the same person, so many sources of uncertainty between instruments can be eliminated. This makes conparison much more reliable. The PTLTP, in adopting a monolithic equal optical path beam splitter, is much more stable than the LTP II, which uses a separated component beam splitter. For precise profile testing on a plane mirror, it is better to align the returning beam along the optical axis to avoid lateral movement, and a penta-prism scan mode is preferred. Methodology improvement is necessary for further comparison measurements.

\section{ACKNOWLEDGMENTS}

The authors would like to thank Peter Takacs helpful discussion and checking article. Thank colleagues of NSRRC for all helps. This research was sponsored in part by the U.S. Department of Energy and the National Synchrotron Radiation Research Center, Taiwan. This manuscript has been authored by Brookhaven Science Associates, LLC under Contract No. DE-AC02-98CH10886 with the U.S. Department of Energy. The United States Government retains, and 
the publisher, by accepting the article for publication, acknowledges, a world-wide license to publish or reproduce the published form of this manuscript, or allow others to do so, for the United States Government purposes. This research was sponsored in part by the U. S. Department of Energy and the National Synchrotron Radiation Laboratory, University of Science and Technology of China.

\section{REFERENCES}

1. Shinan Qian, Peter Takacs, "Portable long trace profiler: concept and solution", Review of Scientific Instruments, 72(8), 3198-3204, (2001)

2. Peter Takacs, Shinan Qian and Jeffrey Colbert, Design of a long trace surface profiler, SPIE Vol. 749, 59-64, Aug. 1987

3. Peter Takacs, Shinan Qian, Surface profiling interferometer, US patent No.U4884697, Dec.5, 1989

4. Shinan Qian, W. Jark, Peter Takacs, "The penta-prism LTP: A long-trace-profiler with stationary optical head and moving penta-prism", Rev. Sci. Instrum., 66, 2562-2569 (1995).

5. Shinan Qian, Peter Takacs, "Equal optical path beam splitter for a pencil beam interferometer and shearing interferometer", Opt. Eng. 42(4), 929-934, (2003)

6. Shinan Qian, Peter Takacs, Beam splitter and method for generating equal optical path length beams, US Patent No. US 6,611,379 B2, Aug. 26, 2003

7. Shinan Qian, Qiuping Wang, Yilin Hong, Peter Takacs, Multiple functions Long Trace Profiler (LTP-MF) for National Synchrotron Radiation Laboratory of China, presented to SPIE $50^{\text {th }}$ annual meeting, 2005 